\title{
Periodic Abstinence From Pap (PAP) Smear Study: Women's Perceptions of Pap Smear Screening
}

Mindy Smith, MD, MS

Linda French, MD

Henry C. Barry, MD, MS

Department of Family Practice, Michigan State University, East Lansing, Mich

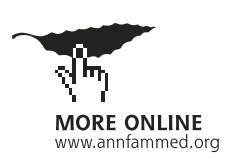

Conflicts of interest: none reported

\section{CORRESPONDING AUTHOR}

Mindy Smith, MD, MS

Department of Family Practice

B-100 Clinical Center

Michigan State University

East Lansing, MI 48824

smithm69@msu.edu

\begin{abstract}
BACKGROUND The purpose of this study was to explore attitudes, beliefs, and perceived barriers to risk-based cervical cancer screening through focus group interviews of patients.

METHODS We conducted 8 focus group interviews of women using semistructured interviews. The investigators independently reviewed the focus group transcripts and identified the overall themes and themes unique to each question using an immersion and crystallization approach.

RESULTS Women are in agreement that cervical cancer screening is important and that women should get Pap smears regularly as an important way of protecting their health. They are not open to the idea of reducing the frequency of Papanicolaou (Pap) smears, however, because they perceive annual screening to be successful in reducing cervical cancer mortality. Additionally, they have concerns about test accuracy. Women are distrustful of the rationale for reducing the frequency of Pap smears. Women's previous bad experiences have reinforced their need for self-advocacy.
\end{abstract}

CONCLUSION Women are reluctant to engage in risk-based cervical cancer screening. In this environment, risk-based cervical cancer screening recommendations are likely to be met with resistance.

Ann Fam Med 2003;1:203-208. DOI: 10.1370/afm.32.

\section{INTRODUCTION}

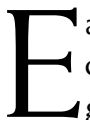
ach year approximately 16,000 women in the United States have cervical cancer diagnosed and about 4,800 die of this disease. ${ }^{1}$ Many groups, including the American Cancer Society ${ }^{2,3}$ and the American College of Obstetricians and Gynecologists, ${ }^{4}$ recommend annual Papanicolaou (Pap) testing, because this practice might have contributed to the declining incidence of invasive cervical cancer during the past 40 years. ${ }^{5}$ Recommendations for Pap smear frequency are in conflict, however. Some groups, including the US Preventive Services Task Force ${ }^{5}$ and the Canadian Task Force, ${ }^{6}$ suggest that low-risk women need Pap smears only every 3 years after 3 consecutive normal Pap smear results. These recommendations are based upon data from 8 cervical cancer screening programs with more than 1.8 million women. ${ }^{7}$ These programs show that the cumulative incidence of invasive cervical cancer was reduced $64.1 \%$ when the interval between Pap tests was 10 years, $83.6 \%$ at 5 years, $90.8 \%$ at 3 years, $92.5 \%$ at 2 years, and $93.5 \%$ at 1 year.

Putting risk-based screening recommendations into practice, however, presents difficulty for health care providers and patients. Inquiring about risk factors, such as sexual habits, is often embarrassing (to providers and patients), and depending upon how the risk factors are assessed, the findings can have questionable validity. ${ }^{8-16}$ Additionally, some risk factors 
(race, for example ${ }^{17}$ ), can be poor predictors compared with other factors (such as number of sexual partners), and no adequate models exist for predicting cervical cancer. Finally, concerns have been raised about compliance with other screening procedures, such as mammography, clinical breast examinations, and fecal occult blood testing, if the frequency of cervical cancer screening is reduced. ${ }^{18-22}$

We designed a study to assess the feasibility of riskbased cervical cancer screening and to develop an instrument to facilitate the assessment of cervical cancer risk factors. If providers are to adopt recommendations for risk-based screening, it is critical to understand the perceptions, barriers, and concerns of the women they serve. Rolnick and colleagues ${ }^{23}$ assessed the perceptions of patients about the frequency of Pap smears. Their study surveyed 673 women in a large health maintenance organization. More than one half of the women did not know that the recommendations for cervical cancer screening had changed. Of these women, $20 \%$ were skeptical and 50\% made negative comments. These data raise concerns about women's willingness to engage in risk-based screening. The purpose of this study was to explore attitudes, beliefs, and perceived barriers to risk-based cervical cancer screening through focus group interviews of patients from a wide range of backgrounds.

\section{METHODS}

\section{Overview}

This study was conducted as part of a larger study to assess the feasibility of risk-based cervical cancer screening and to develop an instrument to facilitate the assessment of cervical cancer risk factors. We conducted focus group interviews with women seeking care from their primary care physician who were enrolled in a study of risk-based cervical cancer screening. We used semistructured interviews to identify important ideas and concerns about implementing riskbased cervical cancer screening. The instruments and procedures used in this study were reviewed and approved by the Michigan State University Committee on Research Involving Human Subjects and the Sparrow Health System Human Subjects Committee.

\section{Subjects and Setting}

We consecutively recruited women older than 18 years from 8 practices located in 3 different settings: urban practices serving a large percentage of indigent women, a university health center serving mostly middle-class women, and a rural family practice residency. These practices in aggregate serve diverse groups of women. For the focus group interviews, we planned to enroll approximately 60 participants to ensure adequate representation from several potential strata. Several institutional review boards approved the study, and we obtained consent from all participants. We included women aged between 18 and 65 years regardless of the reason for their visit. We excluded women who had had a hysterectomy and those with a history of cervical cancer.

\section{Procedures}

For purposes of the larger study, we used a stratified sampling strategy to ensure the recruitment of at least 200 women of minority race from 600 potential subjects. We approached 2,106 consecutive women attending the offices of the participating practices and asked them to complete a brief questionnaire that asked about demographics, smoking status, sexual practices and partners, previous sexually transmitted diseases, and abnormal Pap smear results. After completing the screening questionnaire, the women who met the eligibility criteria listed above $(\mathrm{n}=1,271)$ were asked about their willingness to participate in a focus group. Of the 812 (64\% of those eligible) women who expressed interest in the focus group, we attempted to contact all by telephone with a follow-up mailed invitational letter.

For the focus group interviews, we recruited patients from all 8 participating practices. We invited women consecutively until 20 women agreed to participate in each group. We anticipated that of these 20 women, 6 to 10 would actually attend the session at the specific time and location. We purposefully sampled each stratum to achieve 8 focus groups that were homogeneous for at least one of the following characteristics: age (younger than 40 years and 40 years and older), education (12 years or less and more than 12 years), and race (white and nonwhite). We used 40 years of age as a cutoff because of transitions in competing health issues, including sexually transmitted diseases, reproduction, and other preventive health measures that change around 40 years. We used high school education as a proxy for socioeconomic status and race strata to ease potential communication barriers that might occur within heterogeneous groups.

We held the focus group interviews in locations convenient to each of the practices: local school library, community health center meeting room, and conference rooms at clinical sites. We held each session after normal clinical hours. We compensated the participants for their time and provided food and beverages during the sessions.

A trained African American female moderator conducted the focus group interviews using semistructured methods (described in an online supplemental Appendix at http://www.annfammed.org/cgi/content/ 
full/1/4/203/DC1) based upon the Patient Path Model ${ }^{24}$ and on the Behavioral Change Model. ${ }^{25}$ She began each session with a brief orientation to the topic of health screening. She also developed with the group the rules for conduct of the session that would ensure privacy, respect, and flow of discussion. She then proceeded with specific questions and prompts to explore further issues raised by the group in response to the items.

We made tape recordings of all the sessions, and field notes were taken by a second research associate who was present during the focus group interviews. The field notes summarized major themes from the note taker's perspective and provided the observer's insights into the emotional content, personality, demeanor, and feelings of the focus group participants.

\section{Data Analysis}

We transcribed tape recordings of the focus group interviews. From these transcripts and the notes taken at the time of the focus group interview, the 3 senior investigators independently identified the overall themes and themes unique to each question using an immersion and crystallization approach. ${ }^{26}$ We held meetings with the senior investigators to reach consensus on themes and used the audiotapes to assist in resolving discrepancies. We then categorized the themes and coded the data collected in the focus groups. We had no a priori coding scheme but identified codes from within the transcripts themselves. After summarizing the results, we returned to the field notes to assess the comprehensiveness and accuracy of our data extraction.

\section{RESULTS}

The focus groups included between 4 and 21 women (Table 1). There were 4 groups homogeneous for race (1 including only African American women, 1 with only Hispanic women, and 2 with only white women); 2 groups with women either younger than 40 years or 40 years and older; 4 groups of women with high school or less education, and 4 groups with women who had more than a high school education.

\section{Pap Smear Screening}

Women across all 8 groups were consistent in support of routine cervical cancer screening with Pap smears. Most women in each group believed strongly that yearly screening was important, although not all women were actually screened yearly, and a few had not had a Pap smear within the preceding 3 years. Sample comments from women included: "Well, I know that I have to have one, you know, if it is the difference between life and death ...," and "You cannot tell me one reason that would be good enough to not have one." Reasons provided by women for yearly screening included fear of rapid disease progression, worry about inaccuracy of Pap smears, an appreciation of the decrease in death rates from cervical cancer associated with increases in Pap testing, and a strong conviction that obtaining Pap smears was doing something important for one's health and one's family.

When asked about the possibility of risk-based cervical cancer screening (supplemental Appendix, questions 6 and 7), most women in each group were firmly set against this practice. Phrases such as "Russian roulette" and "feel cheated" were used to describe these convictions. One or two women in each group were willing to consider risk-based screening if advised by their health provider. Only 1 woman, who was willing to consider risk-based screening, obtained Pap smears at a lesser frequency of 2-year intervals.

A few women believed that reducing the frequency of Pap smears was warranted for women who had a hysterectomy, were older, or were not sexually active, but these opinions pro- 
voked a great deal of discussion in support of yearly testing for these groups of women as well. In fact, women, in several different groups independently were in support of increasing testing to every 6 months as women aged or in the presence of risk factors or a previous abnormal Pap smear finding. In support of their position for routine yearly testing, many women related stories about friends or family who had abnormal Pap smear findings or cervical cancer diagnosed.

Women also discussed when it was appropriate to begin screening Pap smears. Some women, across 5 groups and representing different racial mixes and the full spectrum of educational attainment, believed that Pap smears should begin at the onset of menses. Women in 5 of the 8 groups believed that the onset of sexual activity or upon reaching a certain age threshold (16 to 20 years of age, 6 groups) was the optimal time to begin testing

\section{Test Characteristics}

When asked about the perceived accuracy of Pap smears, women in 7 of the 8 groups reported concerns about false-negative and false-positive test results. One woman's concern about the overall accuracy is reflected in the following statement, "Because, sometimes they're right and sometimes they're wrong. You can go in and get a wrong one, and then you're all scared, then like 2 days later, or whatever, you can go in and get a right one. So they're not really adequate." Only 3 women across 3 different groups believed that the test was highly accurate. A few women in each of 4 different groups reported that a normal Pap test result was reassuring about one's general health. One woman said, "I think they are very effective, because they check for more than just cancer. I mean, us women can have yeast infections, and different kinds of things, and those Pap smears, when they check you, they will let you know whatever's wrong with your body. So I think they're very effective." Most women, however, understood that Pap smears for cervical cancer screening represented only one component of preventive health care.

\section{Risk Factors}

Women expressed particular confusion about cervical cancer progression (mentioned in 5 groups), the role of family history (mentioned in 6 groups), and the terminology used to describe abnormalities. For example, several women used such terms as "high normal" smears in describing their own abnormal reports. From the 3 groups of women with more than high school education, human papilloma virus and its association with cervical cancer was mentioned, but these women appeared equally uncertain as to what it meant with respect to screening.
Risk factors for cervical cancer that were mentioned included sexually transmitted diseases, sexual activity, multiple partners, age, smoking, and having a previous abnormal Pap smear finding. There were a number of misconceptions about other potential risk factors. For example, some women also mentioned being overweight and using birth control pills or tampons as potential risk factors.

\section{Barriers, Enablers, and Information Sources}

The women also identified a number of barriers and enablers to obtaining Pap smears, which are summarized in Table 2.

\section{Self Advocacy}

Women from 7 of the 8 groups were quite vocal about taking responsibility for obtaining health information and cancer-screening information and testing. This important self-advocacy theme represented a consensus within the 7 groups and is reflected in the following statements: "I think that sometimes you have to be able to do research on your own. That's the key to me for having good health because you cannot just depend on the doctor to give you all the information."

Some women spoke of the need to work in collaboration with their physician. "But I think we are partners with our doctor ... we know our body better than they do. But they kind of need to hear from us so that they can kind of determine what it is they need to do to help us." Other women, however, reported bad experiences during a particular visit, which resulted in a lack of trust. Others stated a need to be assertive to get the

\begin{tabular}{|lc|}
\hline \multicolumn{2}{|c|}{ Table 2. Barriers and Enablers to Pap Test } \\
Screening Identified by Focus Group Participants \\
\hline Barriers & Enablers \\
\hline Economic, insurance & Physician-based \\
Patient factors & Female \\
Low personal priority & Generalist \\
Fear & Specialist \\
Embarrassment and discomfort & Good skill in communication \\
(the "yucky" factor) & and listening \\
Fatalism & Reminder systems \\
Time/scheduling & Insurance \\
Confidentiality & Office-based \\
Prior sexual abuse & Patient \\
Physician and staff factors & Free clinics, health department \\
Reluctance/not approachable & Contraception prescribing \\
Scheduling & Parent encouragement \\
Time constraints & Public education \\
Lack of continuity & Books \\
& Media \\
\hline Note: the items in this table are presented in no particular order. \\
\hline
\end{tabular}


information that they need from their physicians. Some women believed that doctors might misinform them because of "kickbacks" or money obtained from Pap smear screening.

Some women were suspicious that recommendations for Pap smear screening intervals were driven by organized medicine and the insurance industry, which were not looking out for their interests. "I don't think HMOs are looking for quality. They're looking to save their pocket, and that's a fact." Even when women are in trusting relationships with health providers, a previous experience often resulted in taking greater selfadvocacy roles. "I don't take what my doctor says as gospel because I've had breast cancer and I firmly believe that if I had followed my original doctor's recommendations, I'd be dead now. I learned at that point that you have to take control, do the research and find what's going on."

\section{Powerful Narratives}

Two major themes emerged from the most powerful stories shared by these women: abuse by clinicians and the role of personal experiences with advanced cancers detected at late stages. One woman shared the following story. "I think that a lot of the male men (sic), they don't care if they go in there and take your guts out.... One time, I went in there and he started yelling at me because I moved. ... I jumped because he didn't tell me what he was going to do.... He threw down his utensils and was swearing at me."

Another woman was particularly touched by her experience caring for another. "I worked at hospice and saw this woman on the last day of her life. She had cervical cancer that had spread through her body. That was one of the most horrific things I had ever seen ... she was like 55 or 60 , so ... why risk it? It is not a big deal."

\section{Field Notes}

On review of the field notes, we found that all major themes were addressed in the primary analysis. A few additional triggers for initiating Pap smears were mentioned, including becoming pregnant and discovering menstrual problems. In one group there was a sense from the women that Pap smear examinations were not as comprehensive as they should be. Women in one group were particularly concerned about waiting for their Pap smear results. There was a strong impression from the moderator about the importance of women's stories and their impact on their health concerns and decisions.

\section{DISCUSSION}

The women who participated in these focus group interviews strongly believed that annual (or even more frequent) screening is very important. The messages of the 1960s that, among other things, linked contraception and getting Pap smears have been extremely effective. The habit of having an annual visit to a clinician for a Pap smear appears to be firmly entrenched. We speculate that the years of socialization by the media and various organizations promoting Pap smears as an integral part of women's health care will be difficult to overcome. It is not surprising, then, that women in this study are reluctant to consider risk-based cervical cancer screening. In part, their reluctance appears to be based on a lack of knowledge about the risk factors for cervical cancer, its natural history, and the effectiveness of annual compared with triennial screening.

To overcome the misperceptions and concerns expressed will require considerable education, communication, and reassurance. The women in this study prefer a proactive approach, in part because of mistrust of physicians, test characteristics, and the perceived success of yearly strategies in reducing cervical cancer. Whereas some women were aware of the shifting recommendations for Pap smear screening, they were unaware of the underlying rationale. Women are suspicious that changes in the recommended screening intervals are motivated by economic factors and not by science. Although the discussion included potential health information sources for women, the opposition to less frequent screening prevented the groups from providing strategies to implement risk-based cervical cancer screening.

We were especially impressed with the role of narratives about their personal experiences and those of loved ones in accessing health care. These stories had common themes, including impersonal providers and staff, poor communication, limited access, and abuse. Although these experiences were indirectly related to the research, they were prominent in the women's memories and colored their willingness to change their beliefs or alter their practices.

We identified several limitations in this study. We recruited women who were seeking care from their usual health caregiver and were willing to participate in a study; therefore, their views might not represent those of women in the community. The consistency of themes across groups is striking, however, and these themes are likely to represent important issues for women who are already obtaining care from a health clinician. The group dynamics, while facilitating discussion in some instances, might have suppressed more timid members from contributing, especially as we had groups that were quite large. Although we used an experienced mediator to try to draw out alternate viewpoints, it is possible that some opinions were missed. We held some group sessions that were homogeneous 
and others that were heterogeneous with respect to ethnicity, age, and educational level. We noted no differences in themes across the groups with the exception of issues related to access to health care.

The authors are struck by the parallels of women's reluctance to reduce or abandon screening using a method that is perceived to be highly effective and the secular trends toward the use of more screening modalities without regard to their effectiveness. The Star Trek Tricorder total body evaluation that gives perfect health information is an image we have difficulty abandoning. "I wouldn't mind if there were more screenings. I know they have whole body scans, so I can just stand there and they can tell me if there is anything hiding." In contradistinction, health caregivers have difficulty abandoning questionable practices. Many ineffective modalities, such as the routine antenatal sonogram and electron beam computerized tomography, have reached boutique status because clinicians cannot agree on their appropriate use. It should be no surprise that women are reluctant to reduce the use of Pap smears.

We identified a number of important themes shared by the women in this study about cervical cancer screening. Women are reluctant to risk adversely affecting a successful approach by reducing the frequency of Pap smears. Women are distrustful of the rationale for reducing the frequency of Pap smears, but they are also empowered to get the information they need to make decisions. This latter point provides an opportunity to influence their belief systems. We encourage future investigators and policy makers to include the opinions and concerns of women before or in concert with changing guidelines or in planning future research in this area.

To read commentaries or to post a response to this article, see the online version at http://www.annfammed.org/cgi/content/full/1/4/203.

Key words: Attitude to health; cervix neoplasms/diagnosis; community health care; health promotion; mass screening; Papanicolaou test; practice-based research; public health; qualitative research

Submitted September 19, 2002; submitted, revised, February 12, 2003; accepted March 14, 2003.

Funding support: This work was funded by the Michigan Cancer Consortium Grant: Periodic Abstinence from Pap (PAP) Smear Study: Assessment of Barriers and Measurement of Risk

Acknowledgments: The authors are grateful to the practitioners who allowed us to disrupt their workflow and their staff who made the study run so smoothly. We also thank Pam Patterson for her coordination of the study; Traci Ristau for coordinating the focus groups, transcribing the tapes, and entering data; and Doris Wilkins for her expertise in moderating the focus group interviews. We also are grateful to Nancy Elder, MD, for her advice on improving the manuscript.

\section{References}

1. Wingo PA, Tong T, Bolden S. Cancer statistics, 1995. CA Cancer J Clin. 1995;45:8-30.

2. Mettlin C, Dodd GD. The American Cancer Society guidelines for the cancer-related checkup: an update. CA Cancer J Clin. 1991;41:279-282.

3. American Cancer Society Update January 1992: the American Cancer Society guidelines for the cancer-related checkup [update]. CA Cancer ] Clin. 1992;42:44-45.

4. Committee on Gynecologic Practice. American College of Obstetricians and Gynecologists. ACOG committee opinion. Recommendations on frequency of Pap test screening. Number 152-March 1995. Int J Gynaecol Obstet. 1995;49:210-211.

5. US Preventive Services Task Force. Guide to Cinical Preventive Services: Report of the U.S. Preventive Services Task Force. 2nd ed. Baltimore, Md: Williams \& Wilkins; 1996.

6. Canadian Task Force on the Periodic Health Examination. The periodic health examination: 2. 1987 update. CMAJ. 1988;138:618-626.

7. IARC Working Group on Evaluation of Cervical Cancer Screening Programmes. Screening for squamous cervical cancer: duration of low risk after negative results of cervical cytology and its implication for screening policies. Br Med J. 1986;293:659-664.

8. Siegel K, Krauss BJ, Karus D. Reporting recent sexual practices: gay men's disclosure of HIV risk by questionnaire and interview. Arch Sex Behav. 1994;23:217-230.

9. Wilkinson CE, Peters TJ, Harvey IM, Stott NC. Feasibility, reliability and women's views of a risk scoring system for cervical neoplasia in primary care. Br J Gen Pract. 1994;44:306-308.

10. Brody S. Patients misrepresenting their risk factors for AIDS. Int J STD AIDS. 1995;6:392-398.

11. Padian NS, Aral S, Vranizan K, Bolan G. Reliability of sexual histories in heterosexual couples. Sex Transm Dis. 1995;22:169-172.

12. Shew ML, Remafedi GJ, Bearinger LH, et al. The validity of self-reported condom use among adolescents. Sex Transm Dis. 1997;24:503-510.

13. Latkin CA, Vlahov D. Socially desirable response tendency as a correlate of accuracy of self-reported HIV serostatus for HIV seropositive injection drug users. Addiction. 1998;93:1191-1197.

14. Wiley DJ, Grosser S, Qi K, et al, for the Multicenter AIDS Cohort Study Group. Validity of self-reporting of episodes of external genital warts. Clin Infect Dis. 2002;35:39-45.

15. Harrington KF, DiClemente RJ, Wingood GM, et al. Validity of selfreported sexually transmitted diseases among African American female adolescents participating in an HIVISTD prevention intervention trial. Sex Transm Dis. 2001;28:468-471.

16. Weinhardt LS, Forsyth AD, Carey MP, Jaworski BC, Durant LE. Reliability and validity of self-report measures of HIV-related sexual behavior: progress since 1990 and recommendations for research and practice. Arch Sex Behav. 1998;27:155-180.

17. Bradley CJ, Given CW, Roberts C. Race, socioeconomic status, and breast cancer treatment and survival. J Natl Cancer Inst. 2002;94:490-496.

18. Bourguet CC, Gilchrist VJ, Kandula M. Correlates of screening mammography in a family practice setting. J Fam Pract. 1988;27:49-54.

19. Murata PJ, Li JE. Relationship between Pap smear performance and physician ordering a mammogram. J Fam Pract. 1992;35:644-648.

20. Conry CM, Main DS, Miller RS, Iverson DC, Calonge BN. Factors influencing mammogram ordering at the time of the office visit. J Fam Pract. 1993;37:356-360.

21. Hueston WJ, Stiles MA. The Papanicolaou smear as a sentinel screening test for health screening in women. Arch Intern Med. 1994;154: 1473-1477.

22. Pearlman DN, Rakowski W, Ehrich B. Mammography, clinical breast exam, and pap testing: correlates of combined screening. Am J Prev Med. 1996;12:52-64.

23. Rolnick SJ, LaFerla JJ, Jackson J, Akkerman D, Compo R. Impact of a new cervical pap smear screening guideline on member perceptions and comfort levels. Prev Med. 1999;28:530-534.

24. Pommerenke FA, Dietrich A. Improving and maintaining preventive services. Part 1: applying the patient path model. J Fam Pract. 1992; 34:86-91.

25. Montano DE, Taplin SH. A test of an expanded theory of reasoned action to predict mammography participation. Soc Sci Med. 1991;32:733-741.

26. Crabtree BF, Miller WL. Doing Qualitative Research. 2nd ed. Thousand Oaks, Calif: Sage Publications; 1999. 